\title{
Exact Solutions of a Fractional-Type Differential-Difference Equation Related to Discrete MKdV Equation
}

\author{
İsmail Aslan* \\ Department of Mathematics, İzmir Institute of Technology, Urla, İzmir 35430, Turkey
}

(Received November 15, 2013; revised manuscript received January 20, 2014)

\begin{abstract}
The extended simplest equation method is used to solve exactly a new differential-difference equation of fractional-type, proposed by Narita [J. Math. Anal. Appl. 381 (2011) 963] quite recently, related to the discrete MKdV equation. It is shown that the model supports three types of exact solutions with arbitrary parameters: hyperbolic, trigonometric and rational, which have not been reported before.
\end{abstract}

PACS numbers: 05.45.Yv, 04.20.Jb

Key words: differential-difference equation, lattice equation, extended simplest equation method

\section{Introduction}

Many physical and mathematical models are described by differential-difference equations (DDEs) or lattice equations. Because of their crucial role, quite a few integrable DDEs have been developed since the original work of Fermi, Pasta and Ulam in the 1950s. ${ }^{[1]}$ To make mention of some: the Ablowitz-Ladik lattice equation, ${ }^{[2]}$ the Volterra lattice equation, ${ }^{[3]}$ the discrete sine-Gordon equation, ${ }^{[4]}$ the Toda lattice equation, ${ }^{[5]}$ the discrete $\mathrm{KdV}$ equation, ${ }^{[6]}$ and so on. Whereas difference equations are being fully discretized, DDEs are semi-discretized with some (or all) of their space variables discretized while time is usually kept continuous. Thus, they can be regarded as hybrid systems.

Lately, Narita ${ }^{[7]}$ proposed the differential-difference equation

$$
\dot{v}_{n}=\frac{4\left(v_{n-1}-v_{n+1}\right) v_{n}^{2}}{\left(1+v_{n-1} v_{n}\right)\left(1+v_{n} v_{n+1}\right)},
$$

which can be transformed to the discrete $\mathrm{MKdV}$ equation $^{[8]}$

$$
\dot{u}_{n}=\left(u_{n-1}-u_{n+1}\right)\left(1-u_{n}^{2}\right),
$$

via the real discrete Miura transformation

$$
u_{n}=\frac{1-v_{n} v_{n+1}}{1+v_{n} v_{n+1}}
$$

where $v_{n}(t)=v(n, t)$ is the displacement of the $n$-th particle from the equilibrium position and $n \in Z$. In fact, Eq. (1) is derived from a system of lattice equations related to the self-dual network equations. ${ }^{[9]}$ Moreover, Eq. (1) is called fractional-type in the sense that the right-hand side is a fraction of the dependent variable.

On the other hand, several sophisticated and powerful methods for the analytic investigation of DDEs have been put forward in the last four decades or so. Among those, we can name: Hirota's bilinear method, ${ }^{10]}$ Casoratian technique, ${ }^{[11]}$ homotopy perturbation method, ${ }^{[12]}$
ADM-Padé technique, ${ }^{[13]}$ etc. But, most of the methods are not easy to handle and require a thorough knowledge of the solution procedure one has in mind.

In this study, our attention is focused towards traveling-wave solutions of the newly coined differentialdifference equation (1). To get the desired results we will make use of the extended simplest equation method, ${ }^{[14]}$ which was developed to find an exact solution of a nonlinear ODE in the form $P\left(y, y^{\prime}, y^{\prime \prime}, y^{\prime \prime \prime}, \ldots\right)=0$ where $y=y(z)$ is an unknown function, $P$ is a polynomial in the dependent variable $y$ and its derivatives. To solve the given nonlinear ODE one expands its solutions $y=y(z)$ in a finite series form

$$
y(z)=\sum_{k=0}^{N} A_{k} Y^{k}, \quad A_{k}=\text { const. }, \quad A_{N} \neq 0,
$$

where $Y=Y(z)$ are some special functions. The main idea behind the extended simplest equation method is the assumption that $Y=Y(z)$ are not only some special functions but they are the functions that satisfy some ordinary differential equations which are referred to as the simplest equations. Two main features characterize the simplest equation: First, this is the equation of a higher order than the nonlinear ODE to be solved; Second, the general solution of this equation is known. This means that the exact solutions of the original nonlinear ODE can be expressed by a finite series in the general solution $Y=Y(z)$ of the simplest equation. This approach has been proven to be very powerful in finding traveling-wave solutions for numerous nonlinear evolution equations. In what follows, we present an improvement of this method for DDEs.

\section{Methodology}

The procedure can be summarized as follows. ${ }^{[15]}$ Assume that we have a system of $M$ fractional-type DDEs in the form 


$$
\Delta\left(\boldsymbol{v}_{\boldsymbol{n}+\boldsymbol{p}_{1}}(\boldsymbol{x}), \ldots, \boldsymbol{v}_{\boldsymbol{n}+\boldsymbol{p}_{k}}(\boldsymbol{x}), \ldots, \boldsymbol{v}_{\boldsymbol{n}+\boldsymbol{p}_{1}}^{\prime}(\boldsymbol{x}), \ldots, \boldsymbol{v}_{\boldsymbol{n}+\boldsymbol{p}_{k}}^{\prime}(\boldsymbol{x}), \ldots, \boldsymbol{v}_{\boldsymbol{n}+\boldsymbol{p}_{1}}^{(r)}(\boldsymbol{x}), \ldots, \boldsymbol{v}_{\boldsymbol{n}+\boldsymbol{p}_{k}}^{(r)}(\boldsymbol{x})\right)=0,
$$

where the dependent variable $\boldsymbol{v}_{\boldsymbol{n}}$ have $M$ components $v_{i, \boldsymbol{n}}$ and so do its shifts; the continuous variable $\boldsymbol{x}$ has $N$ components $x_{i}$; the discrete variable $\boldsymbol{n}$ has $Q$ components $n_{j}$; the $k$ shift vectors $\boldsymbol{p}_{i} \in Z^{Q} ;$ and $\boldsymbol{v}^{(r)}(\boldsymbol{x})$ denotes the collection of mixed derivative terms of order $r$. To search for exact solutions of Eq. (4), we first take the wave transformation

$$
\boldsymbol{v}_{\boldsymbol{n}+\boldsymbol{p}_{s}}(\boldsymbol{x})=\boldsymbol{V}_{\boldsymbol{n}+\boldsymbol{p}_{s}}\left(\xi_{\boldsymbol{n}}\right), \quad \xi_{\boldsymbol{n}}=\sum_{i=1}^{Q} d_{i} n_{i}+\sum_{j=1}^{N} c_{j} x_{j}+\zeta, \quad(s=1,2, \ldots, k),
$$

into consideration where the coefficients $c_{1}, c_{2}, \ldots, c_{N}, d_{1}, d_{2}, \ldots, d_{Q}$ and the phase $\zeta$ are all constants. Then, Eq. (4) changes into

$$
\Delta\left(\boldsymbol{V}_{\boldsymbol{n}+\boldsymbol{p}_{1}}\left(\xi_{\boldsymbol{n}}\right), \ldots, \boldsymbol{V}_{\boldsymbol{n}+\boldsymbol{p}_{k}}\left(\xi_{\boldsymbol{n}}\right), \ldots, \boldsymbol{V}_{\boldsymbol{n}+\boldsymbol{p}_{1}}^{\prime}\left(\xi_{\boldsymbol{n}}\right), \ldots, \boldsymbol{V}_{\boldsymbol{n}+\boldsymbol{p}_{k}}^{\prime}\left(\xi_{\boldsymbol{n}}\right), \ldots, \boldsymbol{V}_{\boldsymbol{n}+\boldsymbol{p}_{1}}^{(r)}\left(\xi_{\boldsymbol{n}}\right), \ldots, \boldsymbol{V}_{\boldsymbol{n}+\boldsymbol{p}_{k}}^{(r)}\left(\xi_{\boldsymbol{n}}\right)\right)=0
$$

To obtain an exact solution, a finite expansion in $\psi^{\prime}\left(\xi_{\boldsymbol{n}}\right) / \psi\left(\xi_{\boldsymbol{n}}\right)$ like

$$
\boldsymbol{V}_{\boldsymbol{n}}\left(\xi_{\boldsymbol{n}}\right)=\sum_{l=0}^{m} a_{l}\left(\frac{\psi^{\prime}\left(\xi_{\boldsymbol{n}}\right)}{\psi\left(\xi_{\boldsymbol{n}}\right)}\right)^{l}
$$

is proposed as a (possible) solution of the nonlinear equation under study, where $m$ is a positive integer, $a_{i}$ 's are constants to be determined, $\psi\left(\xi_{\boldsymbol{n}}\right)$ is the general solution of the simplest equation which can be taken as

$$
\psi^{\prime \prime}\left(\xi_{\boldsymbol{n}}\right)+\mu \psi\left(\xi_{\boldsymbol{n}}\right)=0,
$$

where $\mu$ is an arbitrary constant and prime denotes derivative with respect to $\xi_{\boldsymbol{n}}$. The general solution of Eq. (8) is well known to us. Thus, we get the following cases:

$$
\frac{\psi^{\prime}\left(\xi_{\boldsymbol{n}}\right)}{\psi\left(\xi_{\boldsymbol{n}}\right)}=\sqrt{-\mu}\left(\frac{C_{1} \cosh \left(\sqrt{-\mu} \xi_{\boldsymbol{n}}\right)+C_{2} \sinh \left(\sqrt{-\mu} \xi_{\boldsymbol{n}}\right)}{C_{1} \sinh \left(\sqrt{-\mu} \xi_{\boldsymbol{n}}\right)+C_{2} \cosh \left(\sqrt{-\mu} \xi_{\boldsymbol{n}}\right)}\right)
$$

$$
\begin{aligned}
& \mu<0 \\
& \frac{\psi^{\prime}\left(\xi_{\boldsymbol{n}}\right)}{\psi\left(\xi_{\boldsymbol{n}}\right)}=\sqrt{\mu}\left(\frac{-C_{1} \sin \left(\sqrt{\mu} \xi_{\boldsymbol{n}}\right)+C_{2} \cos \left(\sqrt{\mu} \xi_{\boldsymbol{n}}\right)}{C_{1} \cos \left(\sqrt{\mu} \xi_{\boldsymbol{n}}\right)+C_{2} \sin \left(\sqrt{\mu} \xi_{\boldsymbol{n}}\right)}\right), \quad \mu>0 \\
& \frac{\psi^{\prime}\left(\xi_{\boldsymbol{n}}\right)}{\psi\left(\xi_{\boldsymbol{n}}\right)}=\frac{C_{1}}{C_{1} \xi_{\boldsymbol{n}}+C_{2}}, \quad \mu=0
\end{aligned}
$$

where $C_{1}$ and $C_{2}$ are arbitrary constants. By a straightforward calculation, one can get the identity

$$
\begin{aligned}
& \xi_{\boldsymbol{n}+\boldsymbol{p}_{s}}=\xi_{\boldsymbol{n}}+\phi_{s}, \\
& \phi_{s}=p_{s 1} d_{1}+p_{s 2} d_{2}+\cdots+p_{s Q} d_{Q},
\end{aligned}
$$

where $p_{s j}$ is the $j$-th component of the shift vector $\boldsymbol{p}_{s}$. Hence, considering the trigonometric/hyperbolic function identities and using the functions (9) as well as Eq. (10), we derive the uniform shift formulas

$$
\begin{aligned}
\boldsymbol{V}_{\boldsymbol{n}+\boldsymbol{p}_{s}}\left(\xi_{n}\right) & =\sum_{l=0}^{m} a_{l}\left(\frac{\left(\psi^{\prime}\left(\xi_{n}\right) / \psi\left(\xi_{n}\right)\right) \pm \sqrt{-\mu} \tanh \left(\sqrt{-\mu} \phi_{s}\right)}{1 \pm(1 / \sqrt{-\mu}) \tanh \left(\sqrt{-\mu} \phi_{s}\right)\left(\psi^{\prime}\left(\xi_{n}\right) / \psi\left(\xi_{n}\right)\right)}\right)^{l}, \quad \mu<0, \\
\boldsymbol{V}_{\boldsymbol{n}+\boldsymbol{p}_{s}}\left(\xi_{n}\right) & =\sum_{l=0}^{m} a_{l}\left(\frac{\left(\psi^{\prime}\left(\xi_{n}\right) / \psi\left(\xi_{n}\right)\right) \mp \sqrt{\mu} \tan \left(\sqrt{\mu} \phi_{s}\right)}{1 \pm(1 / \sqrt{\mu}) \tan \left(\sqrt{\mu} \phi_{s}\right)\left(\psi^{\prime}\left(\xi_{n}\right) / \psi\left(\xi_{n}\right)\right)}\right)^{l}, \quad \mu>0, \\
\boldsymbol{V}_{\boldsymbol{n}+\boldsymbol{p}_{s}}\left(\xi_{n}\right) & =\sum_{l=0}^{m} a_{l}\left(\frac{\psi^{\prime}\left(\xi_{n}\right) / \psi\left(\xi_{n}\right)}{1 \pm \phi_{s}\left(\psi^{\prime}\left(\xi_{n}\right) / \psi\left(\xi_{n}\right)\right)}\right)^{l}, \quad \mu=0 .
\end{aligned}
$$

Balancing the highest-order derivative term and the highest order nonlinear term(s) in $\boldsymbol{V}_{\boldsymbol{n}}\left(\xi_{\boldsymbol{n}}\right)$ as in the continuous case, the degree $m$ of Eqs. (7) and (11) from Eq. (6) can be easily determined. Because $\boldsymbol{V}_{\boldsymbol{n}+\boldsymbol{p}_{s}}$ can be thought as being of degree zero in $\psi^{\prime}\left(\xi_{n}\right) / \psi\left(\xi_{n}\right)$, the leading terms of $\boldsymbol{V}_{\boldsymbol{n}+\boldsymbol{p}_{s}}\left(\boldsymbol{p}_{s} \neq 0\right)$ will not have any effect on the balancing procedure. Substituting Eqs. (7) and (11) together with Eq. (8) into Eq. (6), equating the coefficients of $\left(\psi^{\prime}\left(\xi_{n}\right) / \psi\left(\xi_{n}\right)\right)^{l}(l=0,1,2, \ldots)$ to zero, we obtain a system of nonlinear algebraic equations from which the undetermined constants $a_{i}, d_{i}, c_{j}$, and $k$ can be explicitly found. Finally, substituting these results into Eq. (7), one can derive varies kind of exact discrete solutions to Eq. (4).

\section{Analysis}

To find possible exact solutions of Eq. (1), we first introduce the transformation

$$
v_{n}=V_{n}\left(\xi_{n}\right), \quad \xi_{n}=d n+k t+\alpha,
$$

where $d$ and $k$ are real parameters to be specified, while $\alpha$ denotes the phase shift. Then substituting Eq. (12) into Eq. (1) gives

$$
k V_{n}^{\prime}=\frac{4\left(V_{n-1}-V_{n+1}\right) V_{n}^{2}}{\left(1+V_{n-1} V_{n}\right)\left(1+V_{n} V_{n+1}\right)},
$$

where prime denotes derivative with respect to $\xi_{n}$. Based on the method described in Sec. 2, Eq. (12) admits a so- 
lution in the form

$$
V_{n}\left(\xi_{n}\right)=a_{0}+a_{1}\left(\frac{\psi^{\prime}\left(\xi_{n}\right)}{\psi\left(\xi_{n}\right)}\right), \quad a_{1} \neq 0,
$$

where $\psi\left(\xi_{n}\right)$ satisfies Eq. (8), while $a_{0}$ and $a_{1}$ are arbitrary constants to be determined at the stage of solving the problem.

\subsection{Hyperbolic Function Solutions}

In case $\mu<0$, we first derive the expressions $V_{n \pm 1}\left(\xi_{n}\right)$ in accordance with Eq. (11a):

$$
V_{n \pm 1}\left(\xi_{n}\right)=a_{0}+a_{1}\left(\frac{\left(\psi^{\prime}\left(\xi_{n}\right) / \psi\left(\xi_{n}\right)\right) \pm \sqrt{-\mu} \tanh (\sqrt{-\mu} d)}{1 \pm(1 / \sqrt{-\mu}) \tanh (\sqrt{-\mu} d)\left(\psi^{\prime}\left(\xi_{n}\right) / \psi\left(\xi_{n}\right)\right)}\right) .
$$

Substituting Eqs. (14) and (15) along with Eq. (8) into Eq. (13), clearing the denominator, setting the coefficients of $\left(\psi^{\prime} / \psi\right)^{l}(l=0,1, \ldots, 6)$ to zero, we derive a system of nonlinear algebraic equations for $a_{0}, a_{1}, d, k$, and $\mu$. Solving the resulting system, we get the solution set (consist of four cases)

$$
a_{0}= \pm \cosh (d \sqrt{-\mu}), \quad a_{1}= \pm \frac{\sinh (d \sqrt{-\mu})}{\sqrt{-\mu}}, \quad k=-\frac{2 \tanh (d \sqrt{-\mu})}{\sqrt{-\mu}},
$$

which yields discrete hyperbolic function solutions to Eq. (1) as

$$
\begin{aligned}
& v_{n, 1}^{ \pm}(t)=\cosh (d \sqrt{-\mu}) \pm \sinh (d \sqrt{-\mu})\left(\frac{C_{1} \cosh \left(\sqrt{-\mu} \xi_{n}\right)+C_{2} \sinh \left(\sqrt{-\mu} \xi_{n}\right)}{C_{1} \sinh \left(\sqrt{-\mu} \xi_{n}\right)+C_{2} \cosh \left(\sqrt{-\mu} \xi_{n}\right)}\right), \\
& v_{n, 2}^{ \pm}(t)=-\cosh (d \sqrt{-\mu}) \pm \sinh (d \sqrt{-\mu})\left(\frac{C_{1} \cosh \left(\sqrt{-\mu} \xi_{n}\right)+C_{2} \sinh \left(\sqrt{-\mu} \xi_{n}\right)}{C_{1} \sinh \left(\sqrt{-\mu} \xi_{n}\right)+C_{2} \cosh \left(\sqrt{-\mu} \xi_{n}\right)}\right),
\end{aligned}
$$

where $\xi_{n}=d n-(2 \tanh (d \sqrt{-\mu}) / \sqrt{-\mu}) t+\alpha$, while $d, \alpha, \mu(<0), C_{1}$ and $C_{2}$ remain arbitrary.

\subsection{Trigonometric Function Solutions}

In case $\mu>0$, we first derive the expressions $V_{n \pm 1}\left(\xi_{n}\right)$ in accordance with Eq. (11b):

$$
V_{n \pm 1}\left(\xi_{n}\right)=a_{0}+a_{1}\left(\frac{\left(\psi^{\prime}\left(\xi_{n}\right) / \psi\left(\xi_{n}\right)\right) \mp \sqrt{\mu} \tan (\sqrt{\mu} d)}{1 \pm(1 / \sqrt{\mu}) \tan (\sqrt{\mu} d)\left(\psi^{\prime}\left(\xi_{n}\right) / \psi\left(\xi_{n}\right)\right)}\right) .
$$

Substituting Eqs. (14) and (19) along with Eq. (8) into Eq. (13), clearing the denominator, setting the coefficients of $\left(\psi^{\prime} / \psi\right)^{l}(l=0,1, \ldots, 6)$ to zero, we derive a system of nonlinear algebraic equations for $a_{0}, a_{1}, d, k$, and $\mu$. Solving the resulting system, we get the solution set (consist of four cases)

$$
a_{0}= \pm \cos (d \sqrt{\mu}), \quad a_{1}= \pm \frac{\sin (d \sqrt{\mu})}{\sqrt{\mu}}, \quad k=-\frac{2 \tan (d \sqrt{\mu})}{\sqrt{\mu}},
$$

which yields discrete trigonometric function solutions to Eq. (1) as

$$
\begin{aligned}
& v_{n, 3}^{ \pm}(t)=\cos (d \sqrt{\mu}) \pm \sin (d \sqrt{\mu})\left(\frac{-C_{1} \sin \left(\sqrt{\mu} \xi_{n}\right)+C_{2} \cos \left(\sqrt{\mu} \xi_{n}\right)}{C_{1} \cos \left(\sqrt{\mu} \xi_{n}\right)+C_{2} \sin \left(\sqrt{\mu} \xi_{n}\right)}\right), \\
& v_{n, 4}^{ \pm}(t)=-\cos (d \sqrt{\mu}) \pm \sin (d \sqrt{\mu})\left(\frac{-C_{1} \sin \left(\sqrt{\mu} \xi_{n}\right)+C_{2} \cos \left(\sqrt{\mu} \xi_{n}\right)}{C_{1} \cos \left(\sqrt{\mu} \xi_{n}\right)+C_{2} \sin \left(\sqrt{\mu} \xi_{n}\right)}\right),
\end{aligned}
$$

where $\xi_{n}=d n-(2 \tan (d \sqrt{\mu}) / \sqrt{\mu}) t+\alpha$, while $d, \alpha, \mu(>0), \quad$ four cases $)$

$C_{1}$, and $C_{2}$ remain arbitrary.

\subsection{Rational Function Solutions}

In case $\mu=0$, we first derive the expressions $V_{n \pm 1}\left(\xi_{n}\right)$ in accordance with Eq. (11c):

$$
V_{n \pm 1}\left(\xi_{n}\right)=a_{0}+a_{1}\left(\frac{\psi^{\prime}\left(\xi_{n}\right) / \psi\left(\xi_{n}\right)}{1 \pm d\left(\psi^{\prime}\left(\xi_{n}\right) / \psi\left(\xi_{n}\right)\right)}\right)
$$

Substituting Eqs. (14) and (23) along with Eq. (8) into Eq. (13), clearing the denominator, setting the coefficients of $\left(\psi^{\prime} / \psi\right)^{l}(l=0,1, \ldots, 5)$ to zero, we derive a system of nonlinear algebraic equations for $a_{0}, a_{1}, d$, and $k$. Solving the resulting system, we get the solution set (consist of

$$
a_{0}= \pm 1, \quad a_{1}= \pm d, \quad k=-2 d,
$$

which yields discrete rational function solutions to Eq. (1) as

$$
\begin{aligned}
& v_{n, 5}^{ \pm}(t)=1 \pm \frac{d C_{1}}{C_{1}(d n-2 d t+\alpha)+C_{2}}, \\
& v_{n, 6}^{ \pm}(t)=-1 \pm \frac{d C_{1}}{C_{1}(d n-2 d t+\alpha)+C_{2}}
\end{aligned}
$$

where $d, \alpha, C_{1}$, and $C_{2}$ remain arbitrary.

\subsection{Some Special Solutions}

As a first example, if we set " $C_{1}=0$ and $C_{2} \neq 0$ " or " $C_{1} \neq 0$ and $C_{2}=0 "$ in Eq. (17), respectively, then we 
get formal solitary wave solutions to Eq. (1) as

$$
\begin{aligned}
v_{n, 7}^{ \pm}(t)= & \cosh (d \sqrt{-\mu}) \pm \sinh (d \sqrt{-\mu}) \\
& \times \tanh \left(\sqrt{-\mu}\left(d n-\frac{2 \tanh (d \sqrt{-\mu})}{\sqrt{-\mu}} t+\alpha\right)\right), \\
v_{n, 8}^{ \pm}(t)= & \cosh (d \sqrt{-\mu}) \pm \sinh (d \sqrt{-\mu}) \\
& \times \operatorname{coth}\left(\sqrt{-\mu}\left(d n-\frac{2 \tanh (d \sqrt{-\mu})}{\sqrt{-\mu}} t+\alpha\right)\right),
\end{aligned}
$$

where $d, \alpha$, and $\mu(<0)$ remain arbitrary (see Fig. 1).

As a second example, if we let " $C_{1} \neq 0$ and $C_{2}=0$ " or " $C_{1}=0$ and $C_{2} \neq 0$ " in Eq. (21) respectively, then we get formal periodic wave solutions to Eq. (1) as

$$
\begin{aligned}
v_{n, 9}^{ \pm}(t)= & \cos (d \sqrt{\mu}) \pm \sin (d \sqrt{\mu}) \\
& \times \tan \left(\sqrt{\mu}\left(d n-\frac{2 \tan (d \sqrt{\mu})}{\sqrt{\mu}} t+\alpha\right)\right), \\
v_{n, 10}^{ \pm}(t)= & \cos (d \sqrt{\mu}) \pm \sin (d \sqrt{\mu}) \\
& \times \cot \left(\sqrt{\mu}\left(d n-\frac{2 \tan (d \sqrt{\mu})}{\sqrt{\mu}} t+\alpha\right)\right),
\end{aligned}
$$

where $d, \alpha$ and $\mu(>0)$ remain arbitrary (see Fig. 2).

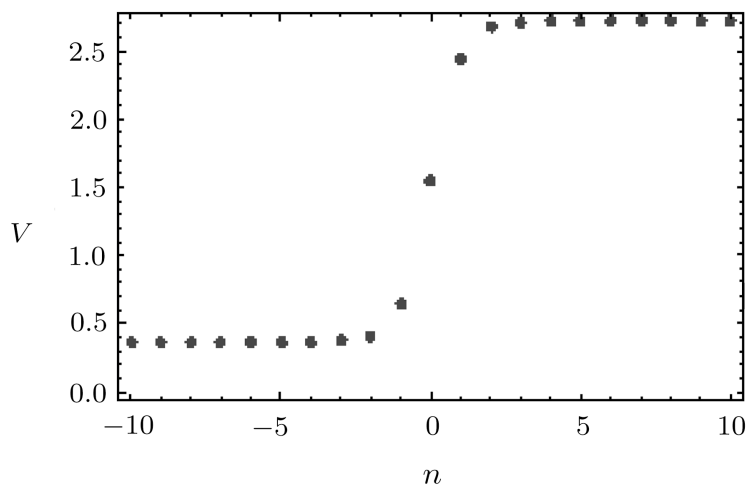

Fig. 1 A profile of $V=v_{n, 7}^{+}(t)$ for $d=1, \mu=-1, \alpha=0$ at time $t=0$ for $n$ from -10 to 10

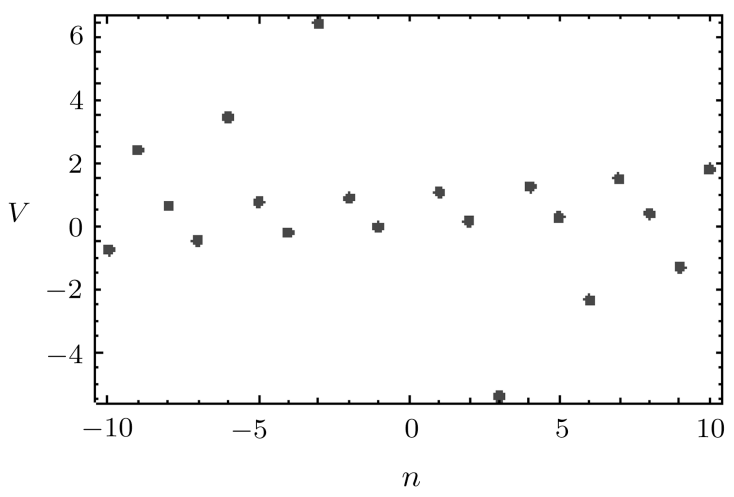

Fig. 2 A profile of $V=v_{n, 10}^{+}(t)$ for $d=1, \mu=1, \alpha=0$ at time $t=0$ for $n$ from -10 to 10 .

As a third example, if we let " $C_{1} \neq 0$ and $C_{2}=0$ " or " $C_{1}=0$ and $C_{2} \neq 0$ " in Eq. (25) respectively, then we get formal rational wave solutions to Eq. (1) as

$$
\begin{aligned}
& v_{n, 11}^{ \pm}(t)=1 \pm \frac{d}{d n-2 d t+\alpha}, \\
& v_{n, 12}^{ \pm}(t)=1,
\end{aligned}
$$

where $d$ and $\alpha$ remain arbitrary. Obviously, Eq. (32) represents a steady-state (time independent) solution (see Fig. 3).

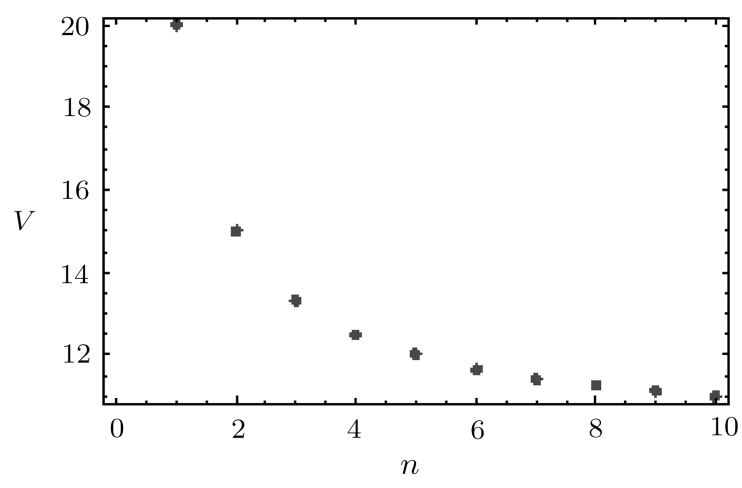

Fig. 3 A profile of $V=v_{n, 11}^{+}(t)$ for $d=1, \alpha=0$ at time $t=0$ for $n$ from 1 to 10 .

Remark At this time, we made a theoretical and computational analysis of Eq. (1) (related to the Korteweg-de Vries equation, which is well known in hydrodynamics) to exhibit wave solutions. Unfortunately, we could not give further details about the real physical meaning of Eq. (1) because of the lack of experimental basis related to it. It is hoped that Eq. (1) might be subject to some adequate physical interpretations in the future. Even though the solutions extracted here belong to only a small set of a large variety of possible solutions for the equation considered, we think that they might serve as seeding solutions for a set of localized structures which can be observed in this equation.

\section{Conclusion}

With the aid of the extended simplest equation method, we successfully analyzed a new lattice equation related to the self-dual network equations. As a result, three types of exact solutions (hyperbolic, trigonometric, and rational) emerged. The obtained results are computed for the first time. Some solutions are analyzed for a particular choice of the parameters. The strength of our technique lies in its ease of use. An advantage of this method is that it avoids tedious algebra and guesswork. Our results could be used as a starting point for numerical procedures as well. It seems that the extended simplest equation method could be implemented to many other fractional-type DDEs. 


\section{References}

[1] E. Fermi, J. Pasta, and S. Ulam, Collected Papers of Enrico Fermi, Chicago University Press, Chicago (1965).

[2] M.J. Ablowitz and J. Ladik, J Math Phys. 16 (1975) 598.

[3] M. Wadati, Prog. Suppl. Theor. Phys. 59 (1976) 36.

[4] S.J. Orfandis, Phys. Rev. D 18 (1978) 3828.

[5] M. Toda, Theory of Nonlinear Lattices, Springer-Verlag, New York (1989).

[6] Y. Ohta and R. Hirota, J. Phys. Soc. Jpn. 60 (1991) 2095.

[7] K. Narita, J. Math. Anal. Appl. 381 (2011) 963.

[8] R. Hirota and J. Satsuma, Progr. Theoret. Phys. Suppl. 59 (1976) 64.
[9] K. Narita, Progr. Theoret. Phys. 106 (2001) 1079.

[10] X.B. Hu and W.X. Ma, Phys. Lett. A 293 (2002) 161.

[11] W.X. Ma and Y. You, Chaos, Solitons \& Fractals 22 (2004) 395.

[12] S.D. Zhu, Y.M. Chu, and S.L. Qiu, Comput. Math. Appl. 58 (2009) 2398.

[13] P. Yang, Y. Chen, and Z.B. Li, Appl. Math. Comput. 210 (2009) 362.

[14] N.A. Kudryashov and N.B. Loguinova, Appl. Math. Comput. 205 (2008) 396.

[15] İ. Aslan, Commun. Nonlinear Sci. Numer. Simul. 15 (2010) 1967. 